\title{
Purification and Partial Characterization of the OmpA Family of Proteins of Pasteurella haemolytica
}

\author{
PARAMESHWAR J. MAHASRESHTI,${ }^{1}$ GEORGE L. MURPHY,${ }^{1}$ JOHN H. WYCKOFF III, ${ }^{2}$ \\ SUSAN FARMER, ${ }^{3}$ ROBERT E. W. HANCOCK, ${ }^{3}$ AND ANTHONY W. CONFER ${ }^{1 *}$ \\ Department of Anatomy, Pathology, and Pharmacology, ${ }^{1}$ and Department of Infectious Disease and Physiology, ${ }^{2}$ \\ College of Veterinary Medicine, Oklahoma State University, Stillwater, Oklahoma 74078, and Department of \\ Microbiology, University of British Columbia, Vancouver, British Columbia, Canada V6T 1Z3 ${ }^{3}$
}

Received 3 June 1996/Returned for modification 16 July 1996/Accepted 22 October 1996

\begin{abstract}
This study was conducted to partially characterize and identify the purity of two major outer membrane proteins (OMPs) (with molecular weights of 32,000 and 35,000 [32K and 35K, respectively]) of Pasteurella haemolytica. The 35K and 32K major OMPs, designated Pasteurella outer membrane proteins A and B (PomA and PomB, respectively), were extracted from $P$. haemolytica by solubilization in $N$-octyl polyoxyl ethylene. The $P$. haemolytica strain used was a mutant serotype A1 from which the genes expressing the 30-kDa lipoproteins had been deleted. PomA and PomB were separated and partially purified by anion-exchange chromatography. PomA but not PomB was heat modifiable. The $\mathrm{N}$-terminal amino acid sequences of the two proteins were determined and compared with reported sequences of other known proteins. PomA had significant $\mathrm{N}$-terminal sequence homology with the OmpA protein of Escherichia coli and related proteins from other gram-negative bacteria. Moreover, polyclonal antiserum raised against the $E$. coli OmpA protein reacted with this protein. PomA was surface exposed, was conserved among $P$. haemolytica biotype A serotypes, and had porin activity in planar bilayers. No homology between the N-terminal amino acid sequence of PomB and those of other known bacterial proteins was found. Cattle vaccinated with live $P$. haemolytica developed a significant increase in serum antibodies to partially purified PomA, as shown by enzyme-linked immunosorbent assays, and to purified PomA and PomB, as detected on Western blots and by densitometry.
\end{abstract}

Bovine pneumonic pasteurellosis, also known as shipping fever pneumonia, is the most common cause of beef cattle losses in North America (55). Pasteurella haemolytica biotype A serotype 1 is the principal etiologic agent of bovine pneumonic pasteurellosis $(17,18)$.

Much effort has been expended towards development of more efficacious vaccines for the prevention of bovine pneumonic pasteurellosis $(11,31)$. To develop such vaccines, investigators have attempted to better understand $P$. haemolytica virulence factors and the host immune response. Recently, Morton et al. (29) demonstrated that cattle vaccinated with $P$. haemolytica A1 outer membranes had enhanced resistance to experimental challenge with the homologous serotype. In a previous study, high antibody responses to $P$. haemolytica saline-extracted proteins with molecular masses of 16, 30, 40, 42, and $86 \mathrm{kDa}$ consistently correlated with resistance to challenge with virulent $P$. haemolytica (32). It was proposed that the protein with a molecular mass of approximately $30 \mathrm{kDa}$ is a major outer membrane protein (OMP) and that this protein may be important in inducing immunity to $P$. haemolytica (13). The tandemly arranged genes for three $P$. haemolytica membrane lipoproteins, which are approximately 28 to $30 \mathrm{kDa}$ and similar to an Escherichia coli $28-\mathrm{kDa}$ lipoprotein, were recently cloned and sequenced $(13,35)$. Serum antibodies to two of the lipoproteins correlated with resistance to experimental challenge $(13,14)$.

Our preliminary studies suggested that another major $P$. haemolytica OMP was similar to the E. coli OmpA protein. The outer membranes of numerous species of gram-negative bac-

\footnotetext{
* Corresponding author. Mailing address: Department of Anatomy, Pathology, and Pharmacology, College of Veterinary Medicine, Oklahoma State University, Stillwater, OK 74078-2007. Phone: (405) 7446744. Fax: (405) 744-5275. E-mail: aconfer@okway.okstate.edu.
}

teria, both enteric and nonenteric, contain major heat-modifiable proteins structurally similar to OmpA (4). Those bacteria include Actinobacillus actinomycetemcomitans (52), Haemophilus somnus (48), Haemophilus influenzae (33, 50), Haemophilus ducreyi (47), Salmonella typhimurium (19), Shigella dysenteriae (5), Neisseria gonorrhoeae (22), Pseudomonas aeruginosa (23, 54), and Serratia marcescens (6). The OmpA protein is important in maintaining integrity of the $E$. coli outer membrane (46), and it stimulates a strong antibody response (41). Also, OmpA may play an important role in virulence, because an OmpA-deficient mutant of $E$. coli $\mathrm{K}-1$ had reduced virulence in an infant rat model of bacteremia (51). Antibodies against OmpA and several OmpA family proteins are bactericidal, opsonic, or protective $(24,25,28,37)$. In contrast, antibodies to the OmpA homolog of $N$. gonorrhoeae inhibit serum bactericidal activity (42).

The aim of the present study was to purify and characterize two $P$. haemolytica OMPs that comigrate with molecular weights of approximately $30,000(30 \mathrm{~K})$ during sodium dodecyl sulfate-12\% polyacrylamide gel electrophoresis (SDS-12\% PAGE) but separate as bands of $35 \mathrm{~K}$ and $32 \mathrm{~K}$ on SDS- $18 \%$ PAGE gels. These proteins were characterized by determination of their N-terminal amino acid sequences and evaluation of antigenicity by Western immunoblotting with sera from vaccinated and subsequently experimentally challenged cattle.

\section{MATERIALS AND METHODS}

Bacteria and culture conditions. $P$. haemolytica $89010807 \mathrm{~N}($ lpp $)$ is a mutant A1 serotype developed by one of us (G.L.M.) (36). The genes for expression of three $30 \mathrm{~K}$ lipoproteins have been detected. This mutant was selected because the $30 \mathrm{~K}$ lipoproteins are in outer and inner membranes and could interfere with purification or detection of OMPs under study. The bacterium was grown for 16 to $18 \mathrm{~h}$ on brain heart infusion (BHI) agar (Difco Laboratories, Detroit, Mich.) containing ampicillin $(10 \mu \mathrm{g} / \mathrm{ml})$ and nalidixic acid $(20 \mu \mathrm{g} / \mathrm{ml})$ to maintain selective pressure. The culture was visually inspected for purity. Several colonies 
were inoculated into $100 \mathrm{ml}$ of BHI broth containing ampicillin $(10 \mu \mathrm{g} / \mathrm{ml})$ and nalidixic acid $(20 \mu \mathrm{g} / \mathrm{ml})$ and incubated at $37^{\circ} \mathrm{C}$ at $80 \mathrm{rpm}$ on a rotary shaker overnight. Subsequently, $100 \mathrm{ml}$ of the overnight culture was inoculated into 2 liters of BHI broth containing ampicillin $(10 \mu \mathrm{g} / \mathrm{ml})$ and nalidixic acid $(20 \mu \mathrm{g} / \mathrm{ml})$ and incubated at $37^{\circ} \mathrm{C}$ for 16 to $18 \mathrm{~h}$ on a rotary shaker at $80 \mathrm{rpm}$. Bacteria were harvested by centrifugation at $8,000 \times g$ for $10 \mathrm{~min}$ at $4^{\circ} \mathrm{C}$, resuspended and washed twice with sterile phosphate-buffered saline (PBS; pH 7.2), and centrifuged as described above to obtain pellets. Cell pellets were stored frozen at $-20^{\circ} \mathrm{C}$ for subsequent OMP extraction.

$P$. haemolytica envelope preparation. Each bacterial pellet from 2 liters of $\mathrm{BHI}$ broth was resuspended in $20 \mathrm{ml}$ of solution containing $10 \mathrm{mM} \mathrm{Na} \mathrm{HPO}_{4}, 5 \mathrm{mM}$ $\mathrm{MgSO}_{4}$ (pH 7.4), DNase (50 $\mu \mathrm{g} / \mathrm{ml}$; Sigma Chemical Co., St. Louis, Mo.), and RNase $(50 \mu \mathrm{g} / \mathrm{ml}$; Sigma Chemical Co.) and sonicated on ice (Branson Sonifier Cell Disruptor; VWR Scientific, Danbury, Conn.) five times for $30 \mathrm{~s}$ each (continuously) with 30 -s intervals with a double-step microtip probe $(50 \mathrm{~W})$ to lyse the cells. The bacterial suspension was centrifuged at $950 \times g$ for $10 \mathrm{~min}$, and the supernatant was collected. The supernatant was then centrifuged at 205,800 $\times g$ for $1 \mathrm{~h}$ at $4^{\circ} \mathrm{C}$ with a $55.2 \mathrm{Ti}$ rotor (Beckman Ultracentrifuge). The envelope-rich pellet was resuspended in $10 \mathrm{ml}$ of distilled $\mathrm{H}_{2} \mathrm{O}$, and the protein concentration was determined by bicinchoninic acid protein assay (Pierce Biochemical).

OMP extraction. Two OMPs designated Pasteurella outer membrane protein A (PomA) (upper band) and PomB (lower band) identified by SDS-PAGE were extracted from the envelope by a modification of a previously described $n$-octyl polyoxyl ethylene (OPOE) (Octyl-POE; Bachem Bioscience Inc., Philadelphia, $\mathrm{Pa}$.) solubilization method (45). The envelope was resuspended by sonicating three times at a concentration of $10 \mathrm{mg} / \mathrm{ml}$ in $10 \mathrm{mM}$ Tris- $\mathrm{HCl}(\mathrm{pH} 8.0)-0.5 \%$ OPOE. After centrifugation at $154,000 \times g$ for $1 \mathrm{~h}$ at $20^{\circ} \mathrm{C}$, the supernatant (designated supernatant 1 ) was collected. The pellet was resuspended by sonication as described above in $10 \mathrm{mM}$ Tris- $\mathrm{HCl}(\mathrm{pH} 8.0)-3 \%$ OPOE. This step was repeated twice to obtain supernatants 2 and 3 . The resulting pellet was then resuspended in $50 \mathrm{mM}$ Tris- $\mathrm{HCl}(\mathrm{pH} 8.0)-50 \mathrm{mM}$ EDTA-3\% OPOE by sonication and centrifuged again as described above. The supernatant was collected (supernatant 4), and the steps were repeated for the remaining pellet to obtain supernatant 5. The resulting pellet was resuspended in $50 \mathrm{mM}$ EDTA-0.4 M $\mathrm{NaCl}-3 \%$ OPOE, sonicated, and centrifuged as described above. Supernatant 6 was collected, and the pellet was resuspended in distilled $\mathrm{H}_{2} \mathrm{O}$, sonicated, and centrifuged as described above. Both the pellet and the supernatant (supernatant 7) were collected. Outer membranes were also extracted separately in $0.5 \%$ sodium $N$-lauryl sarcosine (Sarkosyl; Sigma Chemical Co.) as previously described (29)

Anion-exchange purification. Supernatant 4 (which contained PomA and PomB) was prepared for anion-exchange chromatography by equilibrating in 10 $\mathrm{mM}$ Tricine buffer (buffer A or binding buffer), $\mathrm{pH}$ 8.9, by dialysis. An anionexchange column (Econo-Pac High Q Cartridge; Bio-Rad, Hercules, Calif.) was washed and equilibrated according to the manufacturer's instructions. The sample $(1 \mathrm{ml})$ was applied to the column and washed with $10 \mathrm{ml}$ of buffer A. The column was eluted by a linear gradient of 0.5 to $2 \mathrm{M} \mathrm{NaCl}$ in $10 \mathrm{mM}$ Tricine buffer (elution buffer or buffer B), $\mathrm{pH} 8.9$, at a flow rate of $0.5 \mathrm{ml} / \mathrm{min}$. Fractions containing protein peaks were collected, concentrated by ultrafiltration with a PM 10 filter (Amicon Inc., Beverly, Mass.), and dialyzed against $10 \mathrm{mM}$ Tricine buffer, $\mathrm{pH}$ 8.9. Proteins were identified in peak fractions by SDS-18\% PAGE.

SDS-PAGE. Supernatants and pellets were subjected to SDS-PAGE as described previously $(16,27)$. Briefly, the samples were solubilized in Laemmli sample buffer (27) containing 5\% $\beta$-mercaptoethanol and heated to $100^{\circ} \mathrm{C}$ for 2 to $3 \mathrm{~min}$. Samples were loaded onto a $4.5 \%$ acrylamide stacking gel overlaying an $18 \%$ acrylamide resolving gel (except where stated that a $10 \%$ resolving gel was used) and separated by electrophoresis (Mini Protean II Electrophoresis Cell; Bio-Rad, Rockville Centre, N.Y.). Molecular weights of proteins were determined with known standards.

Anti-PomA sera. Antibodies to PomA were produced by immunizing a 4-weekold Holstein calf with gel-purified PomA as described previously (13). Supernatant 4 was subjected to SDS-PAGE. PomA was identified, excised with a sterile scalpel, and emulsified with an equal volume of Freund's incomplete adjuvant (FIA). The calf was immunized intramuscularly on days 0 and 15 . Sera were collected on days $0,15,28$, and 35 .

ELISA. Sera from the calf immunized with PomA and from cattle described below were assayed for antibody responses to supernatant 6 by an enzyme-linked immunosorbent assay (ELISA) (8). Microtiter plates were coated with supernatant 6 that had been concentrated and dialyzed into coating buffer at a concentration of $50 \mathrm{ng}$ of protein per well. Sera were diluted 1:1,000 and reacted with the antigen. Antibody binding was detected with horseradish peroxidase (HRP)conjugated affinity-purified rabbit anti-bovine immunoglobulin G (IgG) (PelFreez, Rogers, Ark.). All assays were done in triplicate. Data were reported as mean optical densities at $490 \mathrm{~nm}\left(\mathrm{OD}_{490}\right) \pm$ standard deviations.

Western immunoblots. Extracts were subjected to SDS-PAGE, electrophoretically transferred to nitrocellulose membranes as previously described (16), and probed with calf anti-P. haemolytica PomA serum and rabbit anti-E. coli OmpA serum (the latter was kindly provided by Ulf Henning, Tübingen, Germany) (20, 43). The antibody complexes were detected by incubating with HRP-conjugated affinity-purified goat anti-rabbit IgG (Pel-Freez) or HRP-conjugated affinitypurified rabbit anti-bovine IgG (Pel-Freez). Serum samples obtained from cattle vaccinated with $P$. haemolytica (described below) were evaluated on Western immunoblots against PomA and PomB as previously described $(13,14,31,40)$ Briefly, supernatant 6 containing mostly PomA from OPOE extraction and anion-exchange-purified PomB from supernatant 4 were subjected to SDSPAGE, transferred to nitrocellulose membranes, and probed with a 1:25 dilution of cattle serum for $2 \mathrm{~h}$. Immune complexes were detected with the HRP-conjugated rabbit anti-bovine $\operatorname{IgG}(8)$. Color reactions were developed with $0.05 \%$ 4-chloro-1-naphthol dissolved in $20 \mathrm{ml}$ of ice-cold methanol and $0.05 \% \mathrm{H}_{2} \mathrm{O}_{2}$ in $100 \mathrm{ml}$ of Tris-buffered saline, $\mathrm{pH} 7.4$.

Heat modifiability. To determine the heat modifiability of proteins, fractions were solubilized in sample buffer at 37 or $100^{\circ} \mathrm{C}$ for $10 \mathrm{~min}$ and subjected to SDS-PAGE.

Surface exposure of PomA. To determine surface exposure of proteins, $P$. haemolytica cells $(1 \mathrm{ml})$ in the mid-logarithmic phase of growth were collected by centrifugation and washed in an equal volume of enzyme buffer $(0.08 \mathrm{M} \mathrm{Tris-HCl}$ [pH 8.0], $0.1 \mathrm{M} \mathrm{CaCl}_{2}$ ). Cells were resuspended in $0.25 \mathrm{ml}$ of enzyme buffer, and $50 \mu \mathrm{g}$ of chymotrypsin (Sigma) in $0.005 \mathrm{ml}$ of enzyme buffer was added (40). Negative controls received an equal volume of enzyme buffer with no enzyme. Cells were incubated on a rotary shaker $(120 \mathrm{rpm})$ for $1 \mathrm{~h}$ at $37^{\circ} \mathrm{C}$, collected by centrifugation, and washed with $1 \mathrm{ml}$ of PBS. For SDS-PAGE, enzyme-treated and control cells at the same concentration based on $\mathrm{OD}_{650}$ readings were resuspended in $0.05 \mathrm{ml}$ of PBS and $0.05 \mathrm{ml}$ of Laemmli sample buffer and heated at $95^{\circ} \mathrm{C}$ for $5 \mathrm{~min}$ before SDS-10\% PAGE and Western blot analysis (40).

N-terminal amino acid sequencing. Supernatant 4 was subjected to SDSPAGE, electrophoretically transferred to a polyvinylidene difluoride membrane (Bio-Rad, Hercules, Calif.), and stained with Coomassie brilliant blue R-250 (Bio-Rad, Hercules, Calif.). The bands were identified by comparison with molecular weight markers. Strips of membrane containing PomA and PomB were excised and subjected to N-terminal amino acid sequencing (Molecular Biology Resource Facility, University of Oklahoma Health Sciences Center).

Serotype specificity of PomA. To determine the conservation of PomA in different $P$. haemolytica serotypes, whole-cell lysates of A biotypes (serotypes 1 , $2,5,6,7,8,9,10,12,13$, and 14) and strain $89010807 \mathrm{~N}(l p p)$ were solubilized in sample buffer at $100^{\circ} \mathrm{C}$ for $2 \mathrm{~min}$ and subjected to SDS-PAGE. Proteins were transferred to nitrocellulose membranes and probed with a dilution of 1:200 calf anti-PomA serum to detect OmpA family protein bands.

Planar bilayer methods. Planar bilayers were made from $1.5 \%$ oxidized cholesterol (kindly provided by R. Benz, Würzburg, Germany) in $n$-decane. An applied voltage of $50 \mathrm{mV}$ and a bathing solution of $1 \mathrm{M} \mathrm{KCl}$ were employed. The PomA protein was added to the bathing solution on one side of the bilayer to a final concentration of $60 \mathrm{ng} / \mathrm{ml}$. All other experimental conditions were as previously described for outer membrane porin protein $\mathrm{F}$ of $P$. aeruginosa $(\mathrm{OprF})$ (53). Controls were nonporin and standard porin protein preparations from $E$. coli and $P$. aeruginosa.

Antibody responses in cattle. Antibody responses to partially purified PomA (supernatant 6) were quantified by ELISA. Antibody responses to anion-exchange-purified PomA and PomB were quantified on Western immunoblots by using densitometry as used previously in our laboratories $(8,13,14,32,40)$ Serum samples were obtained from 20 weanling cattle that had been previously experimentally vaccinated and challenged with $P$. haemolytica A1 $(9,10,12,39)$. Cattle were equally divided among four groups and vaccinated twice subcutaneously (days 0 and 7) with either PBS, formalin-killed P. haemolytica with aluminum hydroxide (bacterin-AlOH), formalin-killed $P$. haemolytica with FIA (bacterin-FIA), or $10^{9} \mathrm{CFU}$ of live $P$. haemolytica. On day 21 , cattle were challenged transthoracically in the caudal lung lobes with $5 \times 10^{9}$ CFU of $P$. haemolytica (39). Four days later, cattle were euthanatized, and lung lesion scores ranging from 0 to 20 were assigned based on the criteria of intensity and spread of lesion (the higher the score was, the more intense the lesion was) (39).

Reactivity of day 0 and day 21 sera to PomA and PomB on Western immunoblots was quantified by measuring the intensity of the bands with a onedimensional-gel scanner (Protein Database Inc., Huntington Station, N.Y.). The blots were scanned, and the OD (trace OD times millimeters) were determined as indirect measurements of the antibody responses $(8,13,14,32,40)$.

Statistical analysis. Mean values for trace OD times millimeters (mean OD values) for PomA and PomB were compared among the vaccine groups by two-sample $t$ tests (2). Within groups, mean OD values were compared by paired $t$ tests. Linear regression analysis was used to correlate antibody responses as measured by ELISA or Western blot-densitometry and lesion scores (31).

\section{RESULTS}

Protein extraction. Fractionation of $P$. haemolytica $89010807 \mathrm{~N}(l p p)$ by differential solubilization in OPOE detergent resulted in seven supernatant fractions and an insoluble pellet. SDS-PAGE of all the supernatants and the pellet showed that supernatant fractions 4,5 , and 6 contained several membrane proteins with molecular masses ranging from 106 to $18 \mathrm{kDa}$ (Fig. 1). At approximately $35 \mathrm{~K}$, a doublet of bands was seen in fractions 4,5 , and 6 with a progressive decrease in concentration of the lower band. The upper band (designated 


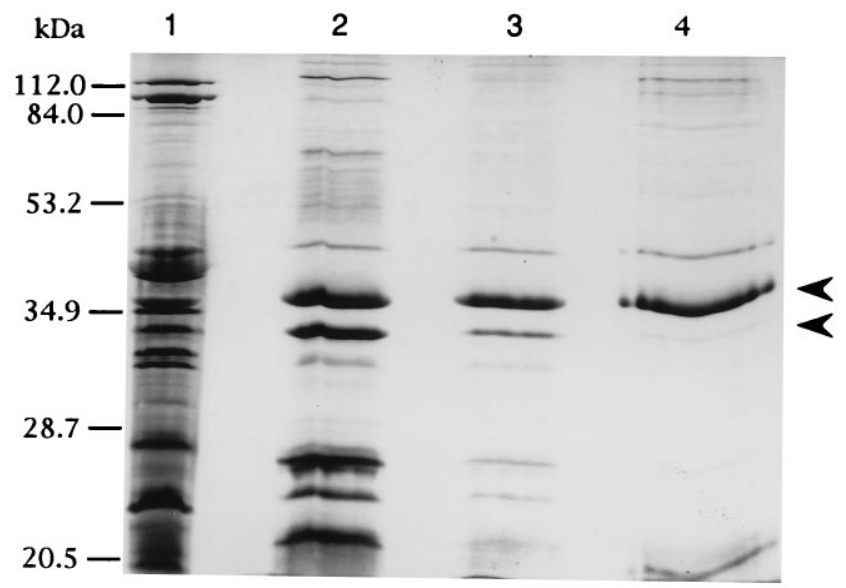

FIG. 1. SDS-PAGE (18\% acrylamide gel, silver stain) showing the OMP bands (including PomA and PomB) of $P$. haemolytica in various OPOE-extracted supernatants and Sarkosyl-extracted OMPs. Lane 1, OMPs of $P$. haemolytica $89010807 \mathrm{~N}$ (lpp); lane 2, supernatant 4; lane 3, supernatant 5; lane 4, supernatant 6. Upper arrowhead, PomA; lower arrowhead, PomB.

PomA) was prominent, and the lower band (designated PomB) was much less so. The apparent molecular weight of PomA and PomB varied with the percentage of the resolving gels used. We observed that PomA and PomB comigrated at approximately $30 \mathrm{~K}$ on $12 \%$ gels. To electrophoretically separate these protein bands, we used SDS-18\% PAGE. This resolved these proteins into two bands corresponding to molecular weights of 35K (PomA) and 32K (PomB) (Fig. 1).

To test whether the two protein bands represented different proteins or were different forms of the same protein, we determined the $\mathrm{N}$-terminal amino acid sequences. This analysis suggested that the two bands represented two different proteins. The N-terminal amino acid sequence of PomA is shown in Table 1. Comparison of this sequence with published sequences by using the BLAST network service (1) revealed that PomA had $60 \%$ identity with the N-terminal amino acid sequence of the E. coli OmpA protein (4) and varied from 60 to $100 \%$ identity with related proteins from several other gramnegative bacteria (Table 1) $(1,5,6,19,33,34,47,52)$.

Further evidence that PomA was related to E. coli OmpA

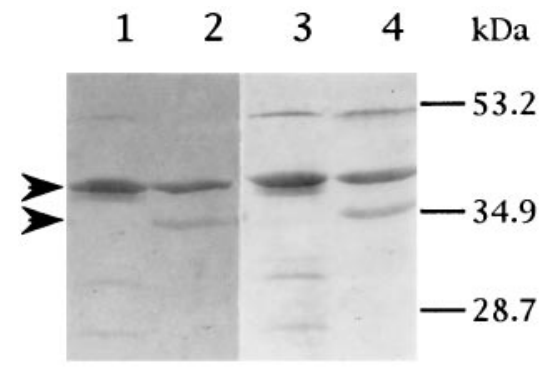

FIG. 2. Western blot showing reactivity of rabbit anti-E. coli OmpA sera and calf anti- $P$. haemolytica (OmpA family protein) PomA sera with PomA of $P$. haemolytica. Lanes 1 and 2, loaded with supernatant 4 (dialyzed against deionized water) solubilized in sample buffer at 100 or $37^{\circ} \mathrm{C}$, respectively, and probed with anti-E. coli OmpA sera (1:200 dilution). (In the dialyzed fraction the PomA is partially heat modified.) Lanes 3 and 4, same as in lanes 1 and 2 except probed with calf anti- $P$. haemolytica OmpA family protein (PomA) serum (1:200 dilution). Upper arrowhead, heat-modified PomA; lower arrowhead, unmodified PomA.

was obtained by Western immunoblot analysis (Fig. 2). Rabbit antiserum against $E$. coli OmpA reacted strongly with PomA of $P$. haemolytica. Calf anti- $P$. haemolytica PomA sera also reacted with the same band.

The molecular weight of $P$. haemolytica PomB was $32 \mathrm{~K}$. The N-terminal amino acid sequence was ADTIG FVDPSYVLENHPVLLDAS. A search of protein sequence databases with the BLAST network service (1) failed to find any substantial homology with other known bacterial proteins.

Heat modifiability of PomA. In undialyzed supernatant 4 or supernatant 6 samples evaluated by SDS-PAGE, PomA had an apparent molecular weight of $35 \mathrm{~K}$ after incubation at 37 and $100^{\circ} \mathrm{C}$. However, SDS-PAGE analysis of PomA present in dialyzed (against deionized water) supernatant 4 fraction and incubated in sample buffer at $37^{\circ} \mathrm{C}$ demonstrated two antigenic bands, one with a molecular weight of $35 \mathrm{~K}$ and the other at 40K (Fig. 2). In contrast, solubilization of dialyzed PomA at $100^{\circ} \mathrm{C}$ for $10 \mathrm{~min}$ resulted in an apparent molecular weight of $40 \mathrm{~K}$. On Western immunoblots, both $35 \mathrm{~K}$ and $40 \mathrm{~K}$ bands were identified with anti-E. coli OmpA and calf anti-P. haemolytica PomA sera, indicating that they represent a single protein. $\mathrm{N}$-terminal amino acid sequencing of both the $35 \mathrm{~K}$ and $40 \mathrm{~K}$ bands demonstrated identical sequences. PomB had the same

TABLE 1. Comparison of N-terminal amino acid sequence of PomA from P. haemolytica A1 with the sequences for $E$. coli OmpA and OmpA-related proteins

\begin{tabular}{|c|c|c|}
\hline Protein (reference[s]) & Sequence & $\%$ Identity \\
\hline Pasteurella haemolytica PomA & APQANTFYAGAKAGWASF HD & \\
\hline Haemophilus influenzae P5 $(1,33)$ & APQENTFYAGYKAGQASFHD & $85.0^{a}$ \\
\hline Haemophilus influenzae fimbrial (1) & APQENTFYAGVKAGQGSFHD & $80.0^{a}$ \\
\hline Enterobacter aerogenes OMP A (1) & APKDNTWYAGGKLGWSQF HD & $65.0^{a}$ \\
\hline Salmonella typhimurium OMP A $(1,19)$ & APKDNTWYAGAKLGWSQYHD & $65.0^{a}$ \\
\hline Shigella dysenteriae OMP A $(1,5)$ & APKDNTWYTGAKLGWSQYHD & $60.0^{a}$ \\
\hline Escherichia coli OMP A $(1,3)$ & APKDNTWYTGAKLGWSQYHD & $60.0^{a}$ \\
\hline Serratia marcescens $(1,6)$ & APKDNTWYTGAKLGWSQYHD & $60.0^{a}$ \\
\hline Actinobacillus actinomycetemcomitans $29 \mathrm{kDa}(51)$ & APQANTFYAGAKA ${ }^{b}$ & $100.0^{d}$ \\
\hline Haemophilus somnus OMP (47) & APQANTFYAGAK ${ }^{C}$ & $100.0^{e}$ \\
\hline Haemophilus ducreyi MOMP (46) & APQADTFYVGAKA ${ }^{b}$ & $83.3^{d}$ \\
\hline
\end{tabular}

${ }^{a}$ Identity of 20 amino acids with the $P$. haemolytica PomA sequence.

${ }^{b}$ Sequence of the next eight amino acids not available.

${ }^{c}$ Sequence of the next nine amino acids not available.

${ }^{d}$ Identity of 13 amino acids with the $P$. haemolytica PomA sequence.

${ }^{e}$ Identity of 12 amino acids with the $P$. haemolytica PomA sequence. 


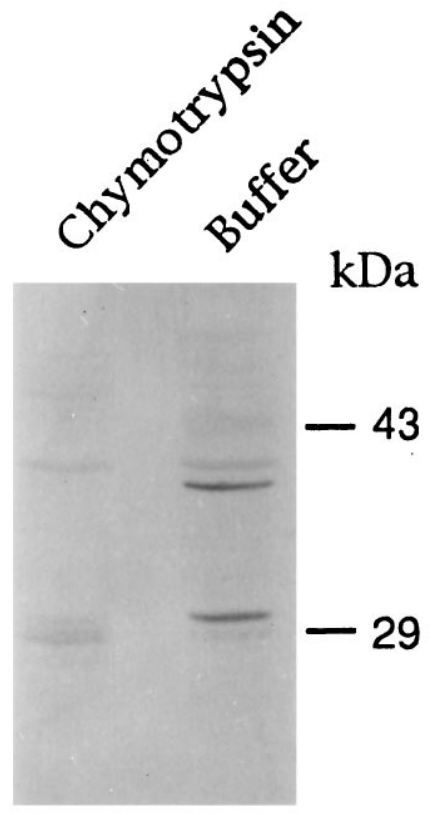

FIG. 3. Western blot showing the surface exposure of PomA protein of $P$. haemolytica. Polyclonal antiserum directed against $E$. coli OmpA was used as the primary antibody. Left lane, whole-cell lysates of chymotrypsin-treated $P$. haemolytica cells showing cleavage of the PomA protein $(\sim 30 \mathrm{kDa}$ on SDS- $-10 \%$ PAGE gels) resulting in loss of immunoreactivity; right lane, whole cells of $P$. haemolytica treated with enzyme buffer only showing intact proteins recognized by $E$. coli OmpA antisera.

molecular weight at both at 37 and $100^{\circ} \mathrm{C}$ in dialyzed and undialyzed preparations (data not shown).

Surface exposure of PomA. Because many of the properties of PomA were similar to those of $E$. coli OmpA, we wanted to know whether PomA was surface exposed like other members of the OmpA family $(7,21,38)$. To determine the presence of surface-exposed regions of PomA, intact $P$. haemolytica cells were treated with chymotrypsin. Whole-cell lysates of protease-treated cells were examined by Western immunoblot analysis with anti-E. coli OmpA serum (Fig. 3). Anti-OmpA serum identified two bands, one at approximately $30 \mathrm{~K}$ and one at $40 \mathrm{~K}$, by SDS-10\% PAGE; the latter most likely was the heat-modified form. Comparison of protease-treated and untreated lysates indicated that chymotrypsin treatment resulted in a loss of immunoreactivity with anti-E. coli OmpA sera (39). This indicated surface exposure of PomA.

Serotype specificity of PomA. On Western immunoblots (Fig. 4) of whole-cell lysates of different serotypes of $P$. haemolytica biotype A, one to two bands were identified with bovine anti-P. haemolytica PomA sera. For serotypes 1 to 8 and 11,13 , and 14, the positive bands corresponded to unmodified (35K) forms of PomA. For serotype 12, a faintly staining band was identified at $35 \mathrm{~K}$ and an intensely staining band was identified at $37 \mathrm{~K}$. Our strain of serotype 9 has a different OMP pattern, and several bands stained positively with anti-PomA serum. This is consistent with the recently published data of Morton et al. (30), which showed that the outer membrane profile of this serotype 9 strain is different from those of other serotypes of $P$. haemolytica biotype A. These results suggest that all $P$. haemolytica biotype A serotypes contain OmpA family proteins.

Anion-exchange purification. Further OPOE extraction of supernatant 4 yielded partial purification of PomA. PomB was greatly reduced in those extractions (supernatants 5 and 6). To determine if PomA and PomB could be separated and each partially purified from supernatant 4 , we used anion-exchange chromatography. All proteins in supernatant 4 bound to the column ( $\mathrm{pH}$ 8.9). Proteins were eluted with a linear gradient of elution buffer resulting in two protein peaks (Fig. 5). These proteins were identified in concentrated and dialyzed peak fractions by SDS-PAGE (Fig. 6). PomB eluted with a salt concentration gradient of 0.62 to $0.66 \mathrm{M} \mathrm{NaCl}$, and PomA eluted with a salt concentration gradient of 1.98 to $2.0 \mathrm{M} \mathrm{NaCl}$. Thus, it was possible to separate and partially purify PomA and PomB effectively by anion-exchange chromatography. N-terminal sequencing confirmed the separation of the two proteins.

Porin activity of PomA. Previous data have indicated that other members of the OmpA superfamily, including OprF (53) and OmpA itself (44), predominantly form small channels of around 300-pS conductance in $1 \mathrm{M} \mathrm{KCl}$. Similar studies with PomA revealed that this protein also formed small ion-permeable channels with a single-channel conductance of $105 \pm 34$ $\mathrm{pS}$ in $1 \mathrm{M} \mathrm{KCl}$. Many of these channels had quite short half-

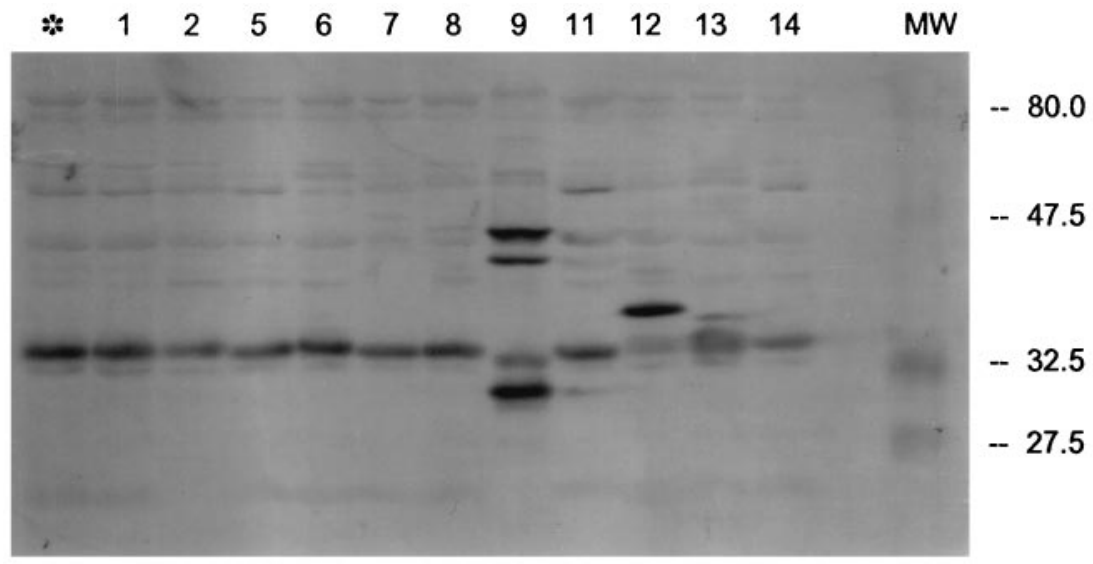

FIG. 4. Western blot showing conservation of OmpA family PomA protein in different $P$. haemolytica A serotypes. The whole-cell lysates of different serotypes and supernatant 4 were subjected to SDS-18\% PAGE, transferred to a membrane, and probed with calf anti-P. haemolytica (OmpA family protein) PomA serum (1:250 dilution). This serum detected the PomA-equivalent bands in all serotypes. The numbers above the lanes represent serotypes. Lane $*, P$. haemolytica $89010807 \mathrm{~N}($ lpp $)$; lane MW, prestained molecular weight markers (marked in thousands on the right). 


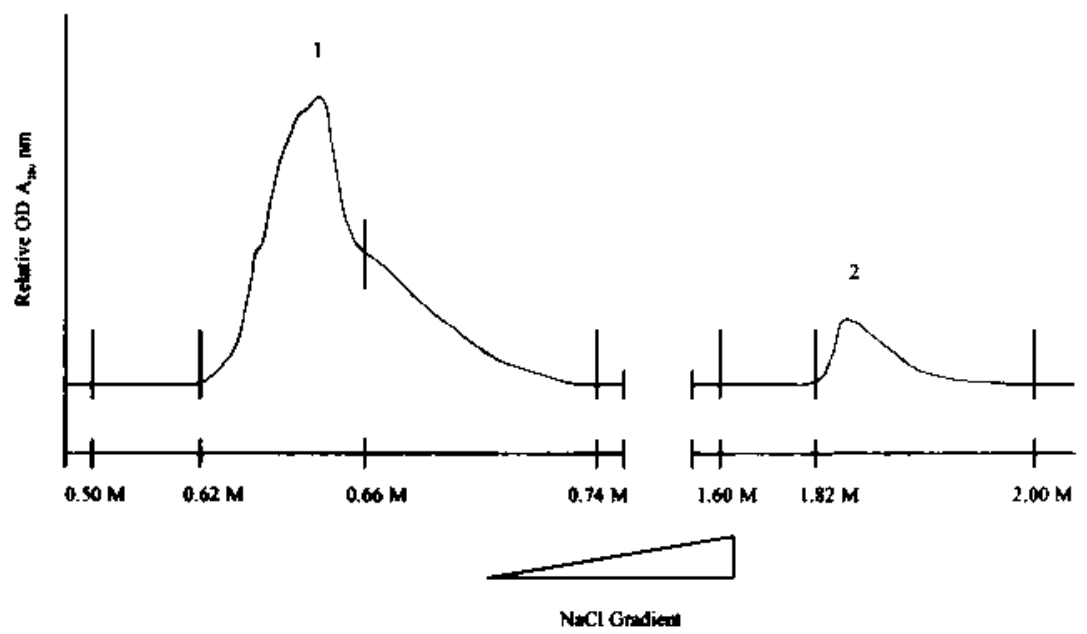

FIG. 5. Anion-exchange purification of PomA (peak 1) and PomB (peak 2) with binding buffer (10 mM Tricine [pH 8.9]) and elution buffer (10 mM Tricine, $2 \mathrm{M}$ $\mathrm{NaCl}[\mathrm{pH} 8.75])$.

lives of around $10 \mathrm{~s}$ or less, and at higher concentrations of protein in the chamber, events as large as $600 \mathrm{pS}$ were observed, possibly due to aggregation or coincident insertion of channels into the bilayer. Thus, these data revealed that PomA, like other members of the OmpA superfamily, had porin activity.

Antibody responses of cattle. To evaluate the antigenicity of Pom A and PomB for cattle, antibody responses (OD values) to supernatant 6, purified PomA, and purified PomB were determined in sera from cattle in four vaccinated groups $(8,13,14$, $32,40)$. There were significant differences between the mean lesion scores for PBS-vaccinated versus live-organism-vaccinated $(P<0.05)$, PBS-vaccinated versus bacterin-FIA-vaccinated $(P<0.05)$, and PBS-vaccinated versus bacterin-AlOHvaccinated $(P<0.05)$ cattle (Table 2$)$. Vaccination of cattle with bacterin-FIA or live bacteria resulted in significant $(P<$ 0.05 ) increases in antibody responses to supernatant 6 as measured by ELISA. Antibody responses did not significantly increase for the PBS and bacterin-AlOH groups. Antibody re-

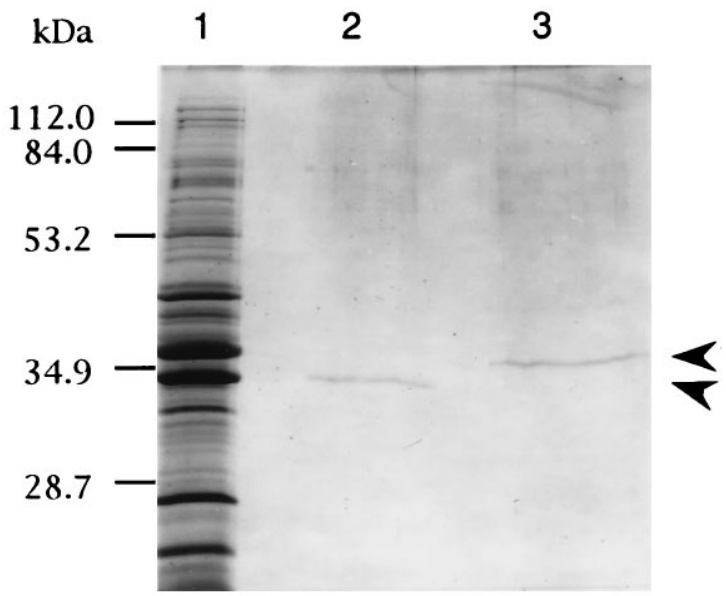

FIG. 6. SDS-18\% PAGE showing different eluted protein peak fractions on anion-exchange chromatography (silver stain). Lane 1 , outer membrane; lane 2, peak 1 containing PomB; lane 3, peak 2 containing PomA. Upper arrowhead, PomA; lower arrowhead, PomB. sponses on day 21 were significantly greater $(P<0.05)$ for the groups vaccinated with bacterin-FIA and live bacteria compared to either the bacterin-AlOH or PBS group.

Antibody responses to purified PomA, as measured by Western blot-densitometry, significantly increased $(P<0.05)$ between days 0 and 21 for all groups. There were no significant differences $(P>0.05)$ between mean antibody responses against purified PomA (day 21) among $P$. haemolytica-vaccinated groups and PBS control groups. The antibody response of vaccinates receiving live bacteria to purified PomB was significantly $(P<0.05)$ greater on day 21 than on day 0 . Significant differences $(P<0.05)$ were seen between antibody responses to PomB (day 21) for groups receiving PBS and live bacteria. There were no significant differences $(P>0.05)$ in antibody responses to PomB between PBS- and bacterin$\mathrm{AlOH}$-vaccinated or PBS- and bacterin-FIA-vaccinated groups.

Regression analysis indicated a strong correlation $(r=$ $-0.645 ; P<0.002$ ) between high antibody responses to supernatant 6 and resistance to challenge. The correlations between high antibody responses to purified PomA and to purified PomB and resistance to challenge were less significant than those to supernatant $6(r=-0.439$ and $r=-0.458$, respectively; $P<0.05$ ).

\section{DISCUSSION}

In this study, we partially purified and characterized two membrane proteins of $P$. haemolytica, termed PomA and PomB (35K and $32 \mathrm{~K}$, respectively), which eluted in fractions 4 , 5 , and 6 of an OPOE OMP extraction. Previous studies (35) identified three lipoproteins $(28$ to $30 \mathrm{kDa})$ that are in the Sarkosyl-soluble and -insoluble fractions of $P$. haemolytica A1. To prevent interference of these proteins in identification and characterization of PomA and PomB, we used P. haemolytica lipoprotein-deficient mutant 89010807N (lpp) (36), which no longer produces these three lipoproteins. Even though supernatant 6 yielded partially purified PomA, it also had relatively small quantities of PomB. Hence, for future studies of the OmpA family protein of $P$. haemolytica, anion-exchange chromatography could be used to separate and purify PomA and PomB from supernatant 4 . 
TABLE 2. Lesion scores and serum antibody responses to supernatant 6 , purified PomA, and purified PomB of $P$. haemolytica for cattle (five per group) vaccinated with various $P$. haemolytica vaccines

\begin{tabular}{|c|c|c|c|c|c|c|c|}
\hline \multirow{3}{*}{ Vaccine group } & \multirow{3}{*}{$\begin{array}{l}\text { Lesion score } \\
\quad(\text { mean } \pm \\
\text { SD })\end{array}$} & \multicolumn{6}{|c|}{ Mean \pm SD antibody response to: } \\
\hline & & \multicolumn{2}{|c|}{ Supernatant $6^{a}$} & \multicolumn{2}{|c|}{$\operatorname{PomA}^{b}$} & \multicolumn{2}{|c|}{ PomB $^{b}$} \\
\hline & & Day 0 & Day 21 & Day 0 & Day 21 & Day 0 & Day 21 \\
\hline PBS & $12.0 \pm 4.1$ & $0.230 \pm 0.211$ & $0.248 \pm 0.099$ & $0.066 \pm 0.147$ & $0.361 \pm 0.113^{d}$ & $0.031 \pm 0.049$ & $0.019 \pm 0.015$ \\
\hline Bacterin- $\mathrm{AlOH}$ & $6.5 \pm 2.4^{c}$ & $0.128 \pm 0.066$ & $0.302 \pm 0.183$ & $0.000 \pm 0.000$ & $0.318 \pm 0.074^{d}$ & $0.026 \pm 0.029$ & $0.090 \pm 0.080$ \\
\hline Bacterin-FIA & $3.8 \pm 4.3^{c}$ & $0.292 \pm 0.377$ & $0.856 \pm 0.342^{c, d, e}$ & $0.000 \pm 0.000$ & $0.458 \pm 0.207^{d}$ & $0.009 \pm 0.013$ & $0.038 \pm 0.037$ \\
\hline Live $P$. haemolytica & $3.6 \pm 2.0^{c}$ & $0.296 \pm 0.186$ & $0.879 \pm 0.327^{c, d, e}$ & $0.040 \pm 0.102$ & $0.520 \pm 0.166^{d}$ & $0.008 \pm 0.015$ & $0.277 \pm 0.180^{c, c}$ \\
\hline
\end{tabular}

${ }^{a}$ Trace $\mathrm{OD}_{490}$ as determined by ELISA.

${ }^{b}$ Trace OD times millimeters, as determined by quantitative Western immunoblot analysis.

${ }^{c}$ Significantly different $(P<0.05)$ from value for PBS group.

${ }^{d}$ Significantly different $(P<0.05)$ from day 0 value.

${ }^{e}$ Significantly different $(P<0.05)$ from value for bacterin-AlOH group.

The N-terminal sequence of PomA and immunoblot analysis data indicate that this protein has substantial homology and shares epitopes with E. coli OmpA. Comparisons of the $\mathrm{N}$ terminal amino acid sequence of PomA with sequences from OmpA family proteins from seven other bacterial species indicated that PomA is probably a member of the OmpA family of proteins. These are major bacterial OMPs and are conserved among many gram-negative bacteria. In addition, immunoreactive OmpA family proteins were found in each of the $P$. haemolytica biotype A serotypes, indicating conservation among $P$. haemolytica serotypes.

The N-terminal amino acid sequences for $P$. haemolytica PomA and PomB were different. We compared the sequence of PomB with other portions of the published sequence of $E$. coli OmpA and found no homology. Therefore, it is unlikely that PomB is a cleaved portion of PomA or a member of the OmpA family of proteins.

The outer membranes of several strains of E. coli, other enteric bacteria, and a variety of nonenteric gram-negative bacteria contain major heat-modifiable proteins similar to OmpA of E. coli (4). Several heat-modifiable proteins have been identified in $P$. haemolytica (26). In supernatant 4 dialyzed against deionized water, PomA appeared in two forms, $35 \mathrm{~K}$ and $40 \mathrm{~K}$, when incubated at $37^{\circ} \mathrm{C}$. Like several other OmpA family proteins, PomA was heat modifiable such that incubation for $10 \mathrm{~min}$ at $100^{\circ} \mathrm{C}$ resulted in only a $40 \mathrm{~K}$ form being present. PomA, however, migrated at $35 \mathrm{~K}$ and was not heat modifiable unless the supernatant was dialyzed against deionized water. This behavior is most likely due to removal of components that stabilize the protein in its heat-unmodified form. Chymotrypsin treatment of $P$. haemolytica indicated that PomA is surface exposed like E. coli OmpA. Functional characterization of PomA revealed that it had porin activity. All these properties of PomA support its inclusion in the OmpA family of proteins.

OmpA proteins confer stability to outer membranes (46), serve as receptors for certain bacteriophages $(15,49)$, and stabilize mating aggregates formed during $\mathrm{F}$ pilus-mediated conjugation (49). A recent study demonstrated that OmpA is a factor in determining resistance to complement-mediated serum killing of a virulent strain of $E$. coli K-1 (51). However, the finding that antibodies to the OmpA family protein in N. gonorrhoeae inhibit serum bactericidal activity indicates that antibodies to this group of proteins could be detrimental to host defenses (42). To determine the potential role of $P$. haemolytica PomA and PomB in immunity, antibody responses to supernatant 6, which contains mainly PomA, and to purified
PomA and PomB were determined in sera from cattle that had been experimentally vaccinated with live or killed $P$. haemolytica. In an ELISA with supernatant 6 (predominately PomA), there were significant increases in antibody response in all $P$. haemolytica vaccinates. Antibody responses in control cattle remained unchanged. As shown by Western blotting-densitometry, antibody responses to purified PomA increased for $P$. haemolytica and for PBS vaccinates. In addition, the correlation between high antibody responses and resistance to challenge was markedly less for the antibodies measured by Western blot-densitometry than for those measured by ELISA. The reasons for these findings are not known with surety. During the course of cattle experiments, one frequently finds an increase in antibodies to $P$. haemolytica due to inhalation of $P$. haemolytica endogenous to the nasal flora. It is unlikely, however, that the increase in antibodies to purified PomA detected in the PBS vaccinates was due to antibodies to endogenous $P$. haemolytica, because the responses to PomB and to supernatant 6 did not increase in that group of cattle.

Other gram-negative bacteria are part of the normal nasal and intestinal flora and could have stimulated antibodies to OmpA family proteins that cross-reacted with PomA. Those could have been detected in sera from the PBS vaccinates. This scenario is possible; however, we found that Western blots against $E$. coli whole-cell lysates using sera from PBS vaccinates had similar reactions to proteins in the $30 \mathrm{~K}$ to $40 \mathrm{~K}$ range for the day 21 and day 0 sera (data not shown). Therefore, it is unlikely that the antibody responses to purified PomA that were detected by Western blotting-densitometry are cross-reactive antibodies to E. coli OmpA.

The results of this experiment raise questions about the potential immunologic significance of antibody responses to PomA. By Western blot-densitometry, the PBS-vaccinated controls had higher quantities of antibodies to PomA but were more susceptible to challenge than the bacterin-AlOH group. A high antibody response to PomA, as measured with these Western blots, was not a good indicator of any potential protection against experimental $P$. haemolytica challenge. However, the potential for cattle to develop antibodies to OmpA family proteins, other than from $E$. coli, and for those antibodies to cross-react with PomA and be detected in Western blots must still be considered. Therefore, characterization of the functional immune response to PomA is needed to determine if PomA has immunologic significance in bovine pneumonic pasteurellosis. 


\section{ACKNOWLEDGMENTS}

This study was funded in part by grant 94-04 from the AVMA Foundation, Oklahoma Agricultural Experimental Station project OKL01438, and a grant from the Medical Research Council of Canada (R.E.W.H).

We thank Sherl Holesko for assistance in typing and graphing.

\section{REFERENCES}

1. Altschul, S. F., W. Gish, W. Miller, E. W. Myers, and D. J. Lipman. 1990. Basic local alignment search tool. J. Mol. Biol. 215:403-410.

2. Bailey, N. T. J. 1981. Statistical methods in biology, p. 77-89. John Wiley, New York, N.Y.

3. Beck, E., and E. Bremer. 1980. Nucleotide sequence of the gene ompA coding the outer membrane protein II of Escherichia coli K-12. Nucleic Acids Res. 8:3011-3024.

4. Beher, M. G., C. A. Schnaitman, and A. P. Pugsley. 1980. Major heatmodifiable outer membrane protein in gram-negative bacteria: comparison with the OmpA protein of Escherichia coli. J. Bacteriol. 143:906-913.

5. Braun, G., and S. T. Cole. 1982. The nucleotide sequence coding for the major outer membrane protein OmpA of Shigella dysenteriae. Nucleic Acids Res. 10:2367-2378.

6. Braun, G., and S. T. Cole. 1984. DNA sequence analysis of the Serratia marcescens ompA gene: implications for the organisation of an enterobacterial outer membrane protein. Mol. Gen. Genet. 195:321-328.

7. Champion, C. I., J. N. Miller, M. A. Lovett, and D. R. Blanco. 1990. Cloning, sequencing, and expression of two class B endoflagellar genes of Treponema pallidum subsp. pallidum encoding the 34.5- and 31.0-kilodalton proteins. Infect. Immun. 58:1697-1704.

8. Confer, A. W., R. D. McCraw, J. A. Durham, R. J. Morton, and R. J. Panciera. 1995. Serum antibody responses of cattle to iron-regulated outer membrane proteins of Pasteurella haemolytica A1. Vet. Immunol. Immunopathol. 47:101-110.

9. Confer, A. W., R. J. Panciera, M. J. Gentry, and R. W. Fulton. 1986. Immunologic response and resistance to experimental pneumonic pasteurellosis in cattle vaccinated with varying doses of lyophilised Pasteurella haemolytica. Am. J. Vet. Res. 47:1853-1857.

10. Confer, A. W., R. J. Panciera, M. J. Gentry, and R. W. Fulton. 1987. Immunologic response to Pasteurella haemolytica and resistance against experimental bovine pneumonic pasteurellosis, induced by bacterins in oil adjuvants. Am. J. Vet. Res. 48:163-168.

11. Confer, A. W., R. J. Panciera, and D. A. Mosier. 1988. Bovine pneumonic pasteurellosis immunity to Pasteurella haemolytica. J. Am. Vet. Med. Assoc. 193:1308-1316.

12. Confer, A. W., R. J. Panciera, and D. A. Mosier. 1986. Serum antibodies to Pasteurella haemolytica lipopolysaccharide: relationship to experimental bovine pneumonic pasteurellosis. Am. J. Vet. Res. 47:1134-1138.

13. Craven, R. C., A. W. Confer, and M. J. Gentry. 1991. Cloning and expression of a $30 \mathrm{kDa}$ surface antigen of Pasteurella haemolytica. Vet. Microbiol. 27:63-78.

14. Dabo, S. M., A. W. Confer, D. Styre, and G. L. Murphy. 1994. Expression, purification and immunological analysis of three Pasteurella haemolytica A1 28-30 kDa lipoproteins. Microb. Pathog. 17:149-158.

15. Datta, D. B., B. Arden, and U. Henning. 1977. Major proteins of the Escherichia coli outer cell envelope membrane as bacteriophage receptors. J. Bacteriol. 131:821-829.

16. Davies, R. L., R. Parton, J. G. Coote, H. A. Gibbs, and J. H. Freer. 1994. Evaluation of different methods for detection of outer membrane proteins and lipopolylaccharides of Pasteurella haemolytica by immunoblotting. J. Immunol. Methods 167:35-45.

17. Frank, G. H. 1986. The role of $P$. haemolytica in the bovine respiratory disease complex. Vet. Med. 81:838-846.

18. Frank, G. H. 1989. Pasteurellosis of cattle, p. 197-222. In C. Adlam and J. M. Rutter (ed.), Pasteurella and pasteurellosis. Academic Press Ltd., London, United Kingdom.

19. Freudl, R., and S. T. Cole. 1983. Cloning and molecular characterization of the omp $A$ gene from Salmonella typhimurium. Eur. J. Biochem. 134:497-502.

20. Freudl, R., G. Braun, I. Hindennac, and U. Henning. 1985. Lethal mutations in the structural gene of an outer membrane protein (ompA) of Escherichia coli K-12. Mol. Gen. Genet. 201:76-81.

21. Freudl, R., S. MacIntyre, M. Degen, and U. Henning. 1986. Cell surface exposure of the outer membrane protein OmpA Escherichia coli K-12. J. Mol. Biol. 188:491-494.

22. Gotschlich, E. C., M. Seiff, and M. S. Blake. 1987. The DNA sequence of the structural gene of gonococcal protein III and the flanking region containing a repetitive sequence. Homology of protein III with enterobacterial OmpA proteins. J. Exp. Med. 165:471-482.

23. Hancock, R. E. W., and A. M. Carey. 1979. Outer membrane of Pseudomonas aeruginosa: heat- and 2-mercaptoethanol-modifiable proteins. J. Bacteriol. 140:902-910.

24. Hughes, E. E., L. B. Gilleland, and H. E. Gilleland. 1992. Synthetic peptides representing epitopes of outer membrane protein F of Pseudomonas aerugi- nosa that elicit antibodies reactive with whole cells of heterologous immunotype strains of $P$. aeruginosa. Infect. Immun. 60:3497-3503.

25. Ichihara, S., M. Hussain, and S. Mizushima. 1981. Characterization of new membrane lipoproteins and their precursors of Escherichia coli. J. Biol. Chem. 256:3125-3129.

26. Knights, J. M., C. Adlam, and P. Owen. 1990. Characterization of envelope proteins from Pasteurella haemolytica and Pasteurella multocida. J. Gen. Microbiol. 136:495-505.

27. Laemmli, U. K. 1970. Cleavage of structural proteins during the assembly of the head of bacteriophage T4. Nature (London) 227:680-685.

28. Mathews, G. J. M., and H. E. Gilleland. 1987. Outer membrane protein F (porin) preparation of Pseudomonas aeruginosa as a protective vaccine against heterologous immunotype strains in a burned mouse model. J. Infect. Dis. 155:1282-1291.

29. Morton, R. J., R. J. Panciera, R. W. Fulton, G. H. Frank, S. A. Ewing, J. T. Homer, and A. W. Confer. 1995. Vaccination of cattle with outer membrane protein-enriched fractions of Pasteurella haemolytica and resistance to experimental challenge. Am. J. Vet. Res. 56:875-879.

30. Morton, R. J., K. R. Simons, and A. W. Confer. 1996. Major outer membrane proteins of Pasteurella haemolytica serovars 1-15: comparison of preparation techniques and surface-exposed proteins on selected serovars. Vet. Microbiol. 51:319-330.

31. Mosier, D. A., A. W. Confer, and R. J. Panciera. 1989. The evolution of vaccines for pneumonic pasteurellosis. Res. Vet. Sci. 47:1-11.

32. Mosier, D. A., K. R. Simons, A. W. Confer, R. J. Panciera, and K. D. Clinkenbeard. 1989. Pasteurella haemolytica antigens associated with resistance to pneumonic pasteurellosis. Infect. Immun. 57:711-776.

33. Munson, R. S., Jr., and D. M. Granoff. 1985. Purification and partial characterization of outer membrane proteins P5 and P6 from Haemophilus influenzae type b. Infect. Immun. 49:544-549.

34. Munson, R. S., Jr., S. Grass, and R. West. 1993. Molecular cloning and sequence of the gene for outer membrane protein P5 of Haemophilus influenzae. Infect. Immun. 61:4017-4020.

35. Murphy, G. L., and L. C. Whitworth. 1993. Analysis of tandem, multiple genes encoding 30-kDa membrane proteins in Pasteurella haemolytica A1. Gene 129:107-111.

36. Murphy, G. L., and L. C. Whitworth. 1994. Construction of isogenic mutants of Pasteurella haemolytica by allelic replacement. Gene 148:101-105.

37. Murphy, T. F., L. C. Bartos, P. A. Rice, M. B. Nelson, K. C. Duda, and M. A. Apicella. 1986. Identification of a 16,600-dalton outer membrane protein on non-typable Haemophilus influenzae as a target for human serum bactericidal antibody. J. Clin. Invest. 78:1020-1027.

38. Murphy, T. F., M. B. Nelson, K. C. Dudas, J. M. Mylotte, and M. A. Apicella. 1985. Identification of a specific epitope of Haemophilus influenzae on a 16,600-dalton outer membrane protein. J. Infect. Dis. 152:1300-1301.

39. Panciera, R. J., R. E. Corstvet, A. W. Confer, and C. N. Gresham. 1984. Bovine pneumonic pasteurellosis: effect of vaccination with live Pasteurella species. Am. J. Vet. Res. 45:2538-2542.

40. Pandher, K., and G. L. Murphy. 1996. Genetic and immunologic analysis of a $38 \mathrm{kDa}$ surface exposed lipoprotein of Pasteurella haemolytica A1. Vet. Microbiol. 51:331-341.

41. Puohiniemi, R., M. Karvonen, J. Vuopio-Varkila, A. Muotiala, I. M. Helander, and M. Sarvas. 1990. A strong antibody response to the periplasmic C-terminal domain of the OmpA protein of Escherichia coli is produced by immunization with purified OmpA or with whole E. coli or Salmonella typhimurium bacteria [sic]. Infect. Immun. 58:1691-1696.

42. Rice, P. A., H. E. Vayo, M. R. Tam, and M. S. Blake. 1986. Immunoglobulin $\mathrm{G}$ antibodies directed against protein III block killing of serum-resistant Neisseria gonorrhoeae by immune serum. J. Exp. Med. 164:1735-1748.

43. Ried, G., R. Koebnik, I. Hindennach, B. Mutschler, and U. Henning. 1994. Membrane topology and assembly of the outer membrane protein OmpA of Escherichia coli K12. Mol. Gen. Genet. 243:127-135.

44. Saint, N., E. De, S. Juliens, N. Orange, and G. Molle. 1993. Ionophore properties of OmpA of Escherichia coli. Biochim. Biophys. Acta 1145:119123.

45. Siehnel, R. J., E. Christine, and R. E. W. Hancock. 1992. Polyphosphateselective porin OprO of Pseudomonas aeruginosa: expression, purification and sequence. Mol. Microbiol. 6:2319-2326.

46. Sonntag, I., H. Schwartz, Y. Hirota, and U. Henning. 1978. Cell envelope and shape of Escherichia coli: multiple mutants missing the outer membrane lipoprotein and other major outer membrane proteins. J. Bacteriol. 136:280-285.

47. Spinola, S. M., G. E. Griffiths, K. L. Shanks, and M. S. Blake. 1993. The major outer membrane protein of Haemophilus ducreyi is a member of the OmpA family of proteins. Infect. Immun. 61:1346-1351.

48. Tagawa, Y., M. Haritani, H. Ishikawa, and N. Yuasa. 1993. Characterization of a heat-modifiable outer membrane protein of Haemophilus somnus. Infect. Immun. 61:1750-1755.

49. Van Alphen, L., L. Havekes, and B. Lugtenberg. 1977. Major outer mem- 
brane protein d of Escherichia coli $\mathrm{K}-12$. Purification and in vitro activity of bacteriophage K3 and F-pilus mediated conjugation. FEBS Lett. 75:285-290.

50. Van Alphen, L., T. Riemens, J. Poolman, and H. C. Zanen. 1983. Characteristics of major outer membrane proteins of Haemophilus influenzae. J. Bacteriol. 155:878-885.

51. Weiser, J. N., and E. C. Gotschlich. 1991. Outer membrane protein A (OmpA) contributes to serum resistance and pathogenicity of Escherichia coli $\mathrm{K}-1$. Infect. Immun. 59:2252-2258.

52. Wilson, M. E. 1991 . The heat-modifiable outer membrane protein of Actinobacillus actinomycetemcomitans: relationship to OmpA proteins. Infect.
Immun. 59:2505-2507.

53. Woodruff, W. A., T. R. Parr, R. E. W. Hancock, L. F. Hanne, T. I. Nicas, and B. H. Iglewski. 1986. Expression in Escherichia coli and function of Pseudomonas aeruginosa outer membrane protein F. J. Bacteriol. 167:473-479.

54. Woodruff, W. A., and R. E. W. Hancock. 1989. Pseudomonas aeruginosa outer membrane protein F: structural role and relationship to the Escherichia coli OmpA protein. J. Bacteriol. 171:3304-3309.

55. Yates, W. D. G. 1982. A review of infectious bovine rhinotracheitis, shipping fever pneumonia and viral bacterial synergism in respiratory disease of cattle. Can. J. Comp. Med. 46:225-263.

Editor: P. E. Orndorff 